\title{
G-IQI-Qualitätsindikatoren in einer Universitätsklinik: Konkrete Qualitätsverbesserung oder ein weiterer Datenfriedhof?
}

\author{
T. Petzold \\ M. Eberlein-Gonska
}

Qualitätsmanagement

Schlüsselwörter

Qualitätssicherung

Berichtswesen

Peer Review

Keywords

quality assurance

reporting system

peer review
Institut

Universitätsklinikum Carl Gustav

Carus an der Technischen Universität Dresden AöR, Zentralbereich Qualitäts- und Medizinisches Risikomanagement

\section{Bibliografie}

Dol 10.1055/s-0031-1286079 Dtsch Med Wochenschr 2011; 136: S50 - (c) Georg Thieme Verlag KG Stuttgart · New York ISSN 0012-0472

\section{Korrespondenz}

Thomas Petzold

Universitätsklinikum Carl Gustav Carus an der Technischen Universität Dresden AöR Zentralbereich Qualitätsund Medizinisches Risikomanagement Fetscherstraße 74 01307 Dresden Tel. 00351/458-4540 eMail thomas.petzold@ uniklinikum-dresden.de
Analyse der IQM-Ergebnisse am Universitätsklinikum Carl Gustav Carus Dresden (UKD), Ableitung von Verbesserungsmaßnahmen und konkrete Umsetzung im Arbeitsalltag $\nabla$

Dass es Verbesserungspotentiale im Gesundheitswesen und damit im Krankenhaus gibt, steht spätestens seit der Veröffentlichung „To Err is Human“ außer Frage [1]. In Deutschland wurde im Jahr 2009 die Initiative Qualitätsmedizin ( $\left.\mathrm{IQ}^{\mathrm{M}}\right)$ gegründet. Ihr Ziel ist es, medizinische Behandlungsabläufe nicht nur kritisch zu hinterfragen und diese mit Hilfe von Qualitätsindikatoren transparent darzustellen, sondern insbesondere die Behandlungsqualität in den beteiligten Krankenhäusern zu verbessern. Die GIQI-Qualitätsindikatoren („German Inpatient Quality Indicators“) bieten dabei den Vorteil, das Potenzial der Patientenbehandlung anhand der Ergebnisqualität zu messen.

Der zentrale Ansatz von $\mathrm{IQ}^{\mathrm{M}}$, die kontinuierliche Verbesserung, richtet sich neben der Durchführung sogenannter Peer Reviews insbesondere nach innen, in die Aufbau- und Ablauforganisation der Kliniken. Jedes an IQ ${ }^{\mathrm{M}}$ teilnehmende Krankenhaus hat sich mit Unterzeichnung des Vertrages dazu bekannt, aktiv mit den Ergebnissen der G-IQI-Qualitätsindikatoren zu arbeiten [2]. Im UKD bedeutet dies, in einem ersten Schritt die als auffällig gekennzeichneten Patientenfälle mit den verantwortlichen Mitarbeitern sorgfältig zu analysieren. Aus der Bewertung leiten sich im 2. Schritt Verbesserungsmaßnahmen in Richtung Codierqualität und insbesondere bezüglich der medizinischen Behandlungsqualität ab. Jede Klinik berichtet über das Ergebnis ihrer Analyse dem Klinikumsvorstand und wird vom Zentralbereich Qualitäts- und Medizinisches Risikomanagement bei der Aufbereitung und Auswertung der Daten unterstützt. Zusätzlich werden mit zahlreichen weiteren Methoden und Instrumenten im UKD medizinische Behandlungsabläufe kritisch hinterfragt.

Eine der ältesten Formen klinischer Qualitätssicherung sind die Morbiditäts- und Mortalitätskonferenzen $(M+M)$. Ihr Stellenwert ist unbestritten. In der Klinik für Viszeral-, Thorax- und Gefäßchirurgie werden regelmäßig anhand von Fallbeispielen Behandlungsabläufe interdisziplinär im Hinblick auf unerwünschte Verläufe diskutiert [3]. Unter der Leitung des Klinikdirektors stellen die behandelnden Assistenzärzte die betreffenden Fälle vor und haben somit die Chance, neben der aktiv betriebenen Qualitätssicherung ihr Wissen und analytisches Denken zu festigen. Dabei stellen die IQM-Ergebnisse eine logistisch einfache und zusätzliche Möglichkeit dar, entsprechende Patientenfälle zu identifizieren. Diese
Methodik wird auch im Rahmen der Morbiditätsund Mortalitätskonferenz am Universitäts GefäßCentrum angewandt. Professionsübergreifend werden dort Patientenfälle diskutiert, die aus den Verfahren der externen Qualitätssicherung - $\mathrm{IQ}^{\mathrm{M}}$ und $\S 137$ SGB V - hervorgehen. Dabei wird im Besonderen auf die Fälle der Initiative Qualitätsmedizin eingegangen, die Bewertungskriterien werden aus dem Peer-Review-Verfahren angewandt. Auf der Basis der Routinedaten kann zudem eine bessere Verbindung zu anderen Kennzahlen des Zentrum-Controllings hergestellt werden. Folglich werden auf diesem Weg die G-IQI-Qualitätsindikatoren als Frühwarnindikatoren für das medizinische Risikomanagement genutzt. Diese Synergie zwischen Qualitäts- und Risikomanagement wird aus Sicht des UKD als äußerst hilfreich und vor allem als sinnvoll angesehen.

Aus dieser Perspektive dienen die G-IQI Qualitätsindikatoren auch als wichtige Information für die Strukturgespräche mit dem Vorstand des UKD. Bei diesen jährlich stattfindenden Gesprächen zwischen den Kliniken und dem Vorstand werden seit 2010 regelmäßig u.a. auch die Qualitätsergebnisse der verschiedenen Kennzahlensysteme sowie aus dem Beschwerdemanagement diskutiert. Die Kliniken haben hier die Möglichkeit, ihr Leistungsspektrum darzustellen und auf Besonderheiten bzw. Auffälligkeiten des letzten Jahres hinzuweisen. Des Weiteren bietet es den Klinikdirektoren die Gelegenheit, einen Ausblick angestrebter Entwicklungen ihrer Klinik im Verbund des UKD darzustellen.

Die Chance, ein vor allem zeitnahes Feedback über die medizinische Behandlungsqualität mit Hilfe von validen Qualitätsindikatoren zu erhalten, wird von den Kliniken des UKD als ausgesprochen positiv bewertet. Dies ist der kritische Erfolgsfaktor für die Nutzung von Qualitätskennzahlen und weckt bei den Ärzten den Anreiz, sich mit den Ergebnissen ihrer Behandlungsqualität zeitnah auseinanderzusetzen. Die Bereitschaft, weiterhin am Verfahren $\mathrm{IQ}^{\mathrm{M}}$ teilzunehmen und dessen Entwicklung aktiv zu fördern, steht somit für das UKD außer Frage.

Autorenerklärung: Die Autoren erklären, dass keine relevanten finanziellen Verbindungen in Bezug auf dieses Manuskript bestehen.

Literatur

1 Kohn LT, Corigan JM, Donaldson MS. (Ed.). To err is human - Building a safer health system. Washington D.C.: Institut of medicine: 2000

2 Kuhlen R, Rink O, Zacher J (Hrsg). Jahrbuch Qualitätsme dizin 2010. Berlin: Initiative Qualitätsmedizin: 2010

3 Orlander JD et al. The Morbidity and Mortality Conference: The Delicate Nature of Learning from Error Washington D.C.: AAMC: 2002 